\title{
Growth of Occult Arteriovenous Malformation After Cerebral Hemorrhage Demonstrated by Serial Magnetic Resonance Imaging
}

\author{
-Case Report-
}

\author{
Takahisa Fuse, Yuji NiWA, Masanari UMEZU, and Kazuo YAMADA* \\ Department of Neurosurgery, National Tohsei Hospital, Shizuoka; * Department of \\ Neurosurgery, Nagoya City University Medical School, Nagoya
}

\begin{abstract}
A 19-year-old male presented with sudden onset of right hemiparesis caused by left cerebral hemorrhage. Cerebral angiography demonstrated no vascular abnormality, and the hematoma was removed surgically. At operation, no abnormal vascular lesion was found in the brain adjoining the hematoma. Two years later, magnetic resonance (MR) imaging demonstrated a few foci of flow voids adjacent to the hematoma cavity. Four years after the hemorrhage, MR imaging showed more extensive flow-void abnormalities that indicated growth of an occult arteriovenous malformation (AVM). Cerebral angiography indicated a definite AVM supplied mainly by branches of the middle cerebral artery. Total resection of this lesion was performed. The histological diagnosis was typical AVM. Immunohistochemistry with vascular endothelial growth factor showed staining in the walls of the abnormal vessels. Serial MR imaging is very useful for the diagnosis and management of occult AVMs.
\end{abstract}

Key words: cerebral hemorrhage, occult arteriovenous malformation, vascular endothelial growth factor

\section{Introduction}

Arteriovenous malformations (AVMs) of the brain are generally considered to be congenital malformations that arise during the early fetal stage when newly formed blood vessels begin to differentiate into primitive arteries, capillaries, and veins. ${ }^{10)}$ Growth of an occult AVM and final manifestation as cerebral hemorrhage has been reported in several cases. ${ }^{2,4,5,8,12)}$ Diagnosis has required serial conventional angiography until very recently. We describe a case of occult AVM detected by serial magnetic resonance (MR) imaging over 4 years following cerebral hemorrhage.

\section{Case Presentation}

A 19-year-old male was found unconscious at home and was transported to our hospital by ambulance. On admission, he was semicomatose with mild right

Received August 14, 2000; Accepted November 27, 2000 hemiparesis. Computed tomography showed a subcortical hematoma with ventricular extension on the left (Fig. 1A). Carotid or vertebral angiography showed no abnormality at that time (Fig. 1B, C). Left front temporal craniotomy was performed, and the hematoma was evacuated. No vascular malformation was found despite careful inspection. Cerebral angiography was repeated on the 21st day after onset, but failed to show any vascular abnormalities. After discharge, the patient was in good health and had no neurological deficit.

Serial follow-up MR imaging initially was normal except for the presence of hematoma cavity (Fig. 2A). Two years after the hemorrhage, MR imaging demonstrated a few foci of flow voids adjacent to the hematoma cavity (Fig. 2B). Two years later, MR imaging showed more extensive flow-void abnormalities, indicating growth of an occult AVM not evident at the operation 4 years earlier (Fig. 2C). Cerebral angiography confirmed presence of an AVM supplied mainly by branches of the middle cerebral artery (Fig. 3). We decided to remove the AVM to prevent additional hemorrhagic episodes. 

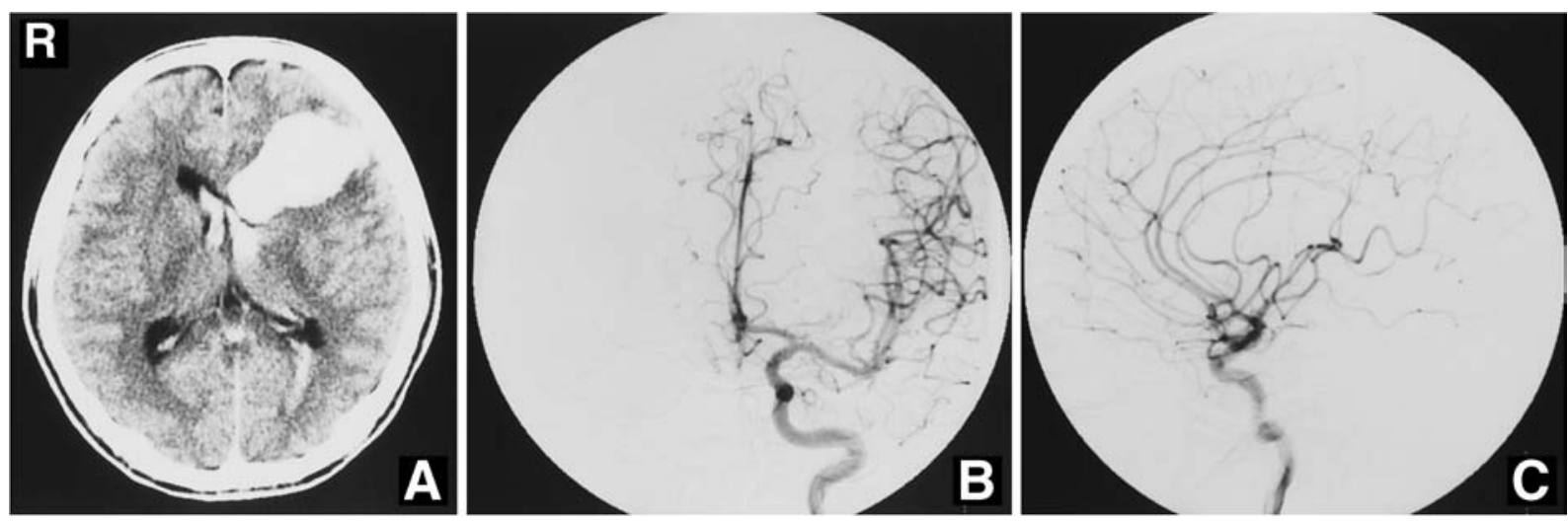

Fig. 1 Radiologic findings on the first hospital day. A: Computed tomography scan showing a subcortical hematoma with intraventricular extension on the left. B, C: Left carotid angiograms, anteroposterior (B) and lateral views (C), demonstrating no abnormal vascular lesions.
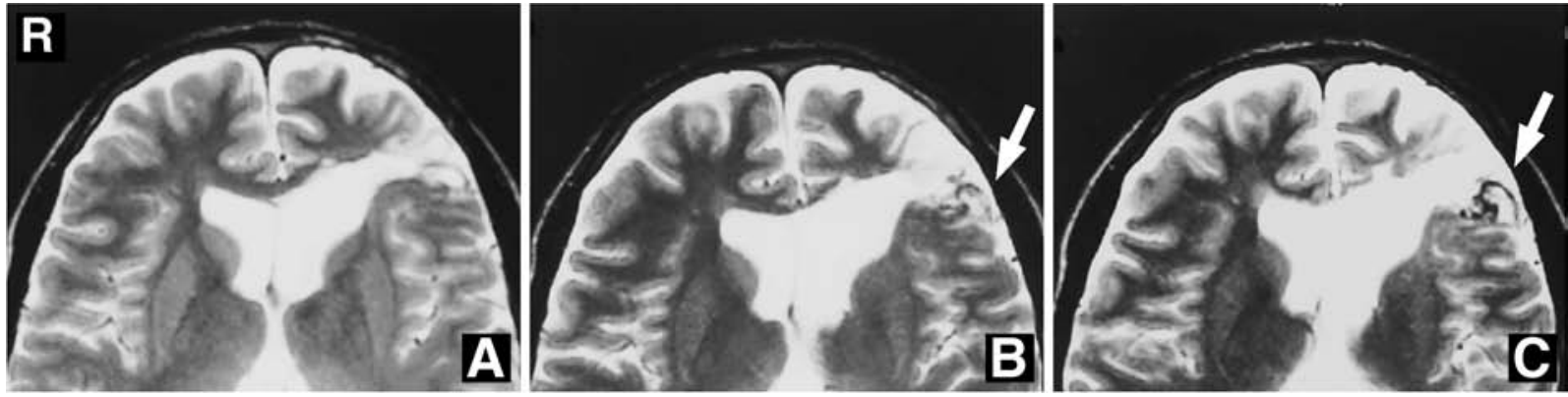

Fig. 2 Serial magnetic resonance (MR) images. A: One month after cerebral hemorrhage, $T_{2^{-}}$ weighted MR image showing no abnormalities except for the hematoma cavity. B: Two years after hemorrhage, MR image showing focal areas of flow voids adjacent to the hematoma cavity, suggesting the presence of a previously occult arteriovenous malformation (AVM) (arrow). C: Four years after hemorrhage, MR image showing more extensive flow-void abnormalities, indicating growth of the AVM (arrow).

Left frontal craniotomy was performed. The abnormal vessels were identified near the premotor area, and the nidus of the AVM was totally removed. During this procedure, the hematoma cavity was not entered. After surgery, the patient suffered a generalized convulsion, with gradual resolution of the postictal state. The patient was discharged with no neurological deficit. Postoperative MR imaging showed no abnormal vascular lesion.

Histological examination of the surgical specimen showed typical cerebral AVM (Fig. 4A). Immunostaining of the surgical specimen using antivascular endothelial growth factor (VEGF) antibody (Oncogene Science, Cambridge, Mass., U.S.A.) showed staining of several abnormal vessel walls (Fig. 4B). Immunohistochemical protocol with omis-

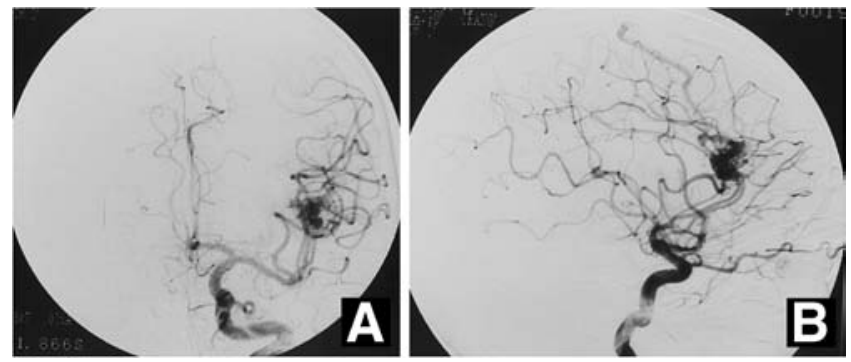

Fig. 3 Left carotid angiograms, anteroposterior (A) and lateral views (B), at the second admission showing the arteriovenous malformation supplied mainly by branches of the middle cerebral artery. 

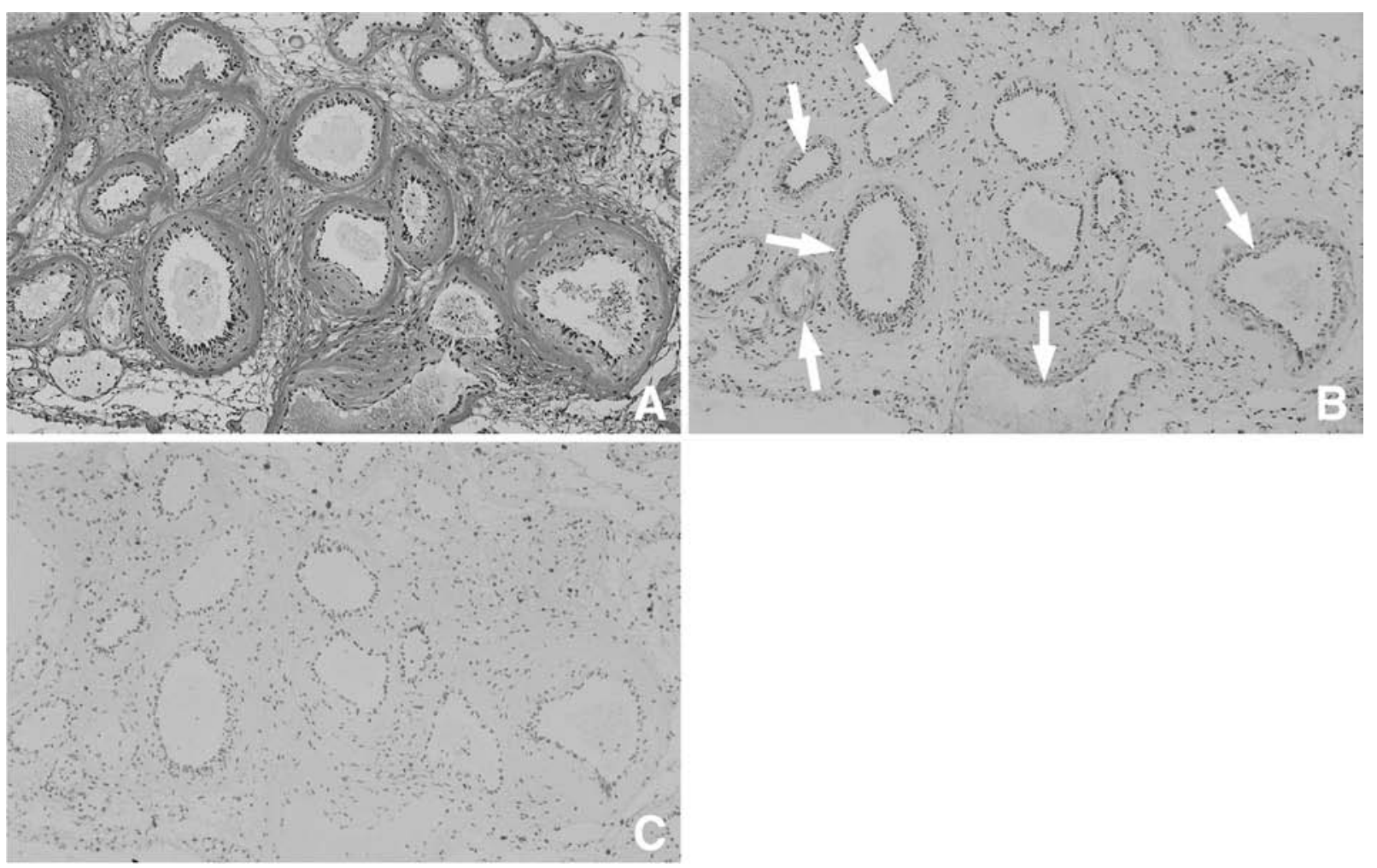

Fig. 4 Photomicrographs of the resected specimen. A: Typical findings of arteriovenous malformation. HE stain, original magnification $\times 100$. B: Immunoreactivity for vascular endothelial growth factor (VEGF) is present in the abnormal blood vessel walls (arrows). The endothelium is the most reactive layer. Anti-VEGF immunostain, original magnification $\times$ 100. C: Control section from the same region as in $B$, showing absence of reaction product when the primary antibody was omitted from the immunostaining protocol. Original magnification $\times \mathbf{1 0 0}$.

sion of the primary antibody showed no staining of adjacent control sections (Fig. 4C).

\section{Discussion}

Initial failure of angiography to detect an AVM responsible for intracerebral hemorrhage can be attributed to small size of the AVM; occlusive pressure from the hematoma; thrombosis caused by compression from the hematoma or posthemorrhagic edema; and vascular spasm, or regressive changes of the vessel wall.4) In the present case, no AVM was identified shortly after cerebral bleeding.

Importantly, small AVMs are considered more likely to grow than large ones. ${ }^{11)}$ Possible mechanisms of occult AVM enlargement following hemorrhage include hemodynamic stress favoring enlargement of fistulous shunts and poorly differentiated vessels; repeated "silent" hemorrhage resulting progressive vascular dilation; and space available for AVM enlargement in the hematoma cavity. ${ }^{10)}$ In our patient, MR imaging 2 years after intracranial hemorrhage demonstrated a few patent abnormal vessels. Repeated silent hemorrhagic episodes are unlikely as a mechanism of growth in this case because serial MR imaging demonstrated no new hemorrhagic change. Therefore, we suspect that a small AVM initially present caused the subcortical hemorrhage and then disappeared, possibly because of thrombosis related to the hematoma. Subsequent ongoing hemodynamic stress at the site of the lesion resulted in regrowth of the AVM. The presence of the residual cavity from the hematoma might also have encouraged expansion of the AVM.

Histological abnormalities characterized by increased vascularity have recently been linked to the angiogenic trophic factor VEGF.6) Immunohistochemistry in the surgical specimen of our case demonstrated vessel wall staining with VEGF, suggesting a contribution to growth of this occult AVM. VEGF and VEGF messenger ribonucleic acid are upregulated in the cells of brain parenchyma 
adjacent to AVMs and in the AVMs. ${ }^{1)}$ VEGF was expressed in four recurrent pediatric $A V M s,{ }^{9)}$ and 30 of 34 cases of AVMs were reactive to VEGF antibody and the endothelium was the most reactive layer of the AVM vessels. ${ }^{3)}$ These recent observations indicate the involvement of angiogenic factor VEGF in the pathogenesis of AVM. The AVM wall may have a biologically changing molecular structure because of continuing angiogenesis, which may be part of the natural history of AVMs. ${ }^{3)}$ Further investigation is necessary to understand how VEGF may induce growth of an occult AVM.

Conventional cerebral angiography remains the gold standard for the diagnosis of AVM. ${ }^{7}$ However, cerebral angiography is associated with an incidence of neurological complications of approximately $0.5 \%$ to $1 \% .{ }^{7)} \mathrm{MR}$ imaging is safe and noninvasive, and can demonstrate a patent $A V M$ as a flow-void signal abnormality. Unfortunately, single MR imaging examination performed for the diagnosis of AVM detects only about $80 \%$ of cases. ${ }^{7)}$

Follow-up examination using serial MR imaging is important in young patients with cerebral hemorrhage of unknown origin, even if angiography following hematoma evacuation demonstrates no vascular lesion. We believe that serial MR imaging can accurately demonstrate patency and enlargement of a suspected occult AVM.

\section{References}

1) Hatva E, Jaaskelainen J, Hirvonen $H$, Alitalo $K$, Halatia M: Tie endothelial cell-specific receptor tyrosine kinase is upregulated in the vasculature of arteriovenous malformations. J Neuropathol Exp Neurol 55: 1124-1133, 1996

2) Isayama $Y$, Nakagawara J, Takeda R, Wada K, Hyogo T, Sasaki T, Nakamura J, Suematsu K: [A case of cerebral arteriovenous malformation revealed at repeated subcortical hematoma with initially normal angiogram]. No Shinkei Geka 19: 1175-1180, 1991 (Jpn, with Eng abstract)

3) Kilic T, Pamir MN, Kullu S, Eren F, Ozak MM, Black PM: Expression of structural proteins and angioge- nic factors in cerebrovascular anomalies. Neurosurgery 46: 1179-1192, 2000

4) Morioka T, Nishino S, Hikita T, Cung LH, Soejima T: Marked growth of an angiographically occult arteriovenous malformation: case report. Neurosurgery 23: 101-103, 1988

5) Peeters FLM: Angiographically demonstrated large vascular malformation in a patient with a normal angiogram 23 years before: a case report. Neuroradiology 23: 113-114, 1982

6) Plate $\mathrm{KH}$, Breier G, Weich HA, Risau W: Vascular endothelial growth factor is a potential tumor angiogenesis factor in human gliomas in vivo. Nature 359: 845-848, 1992

7) Pollock BE, Kondziolka D, Flickinger JC, Patel AK, Bissonette DJ, Lunsford LD: Magnetic resonance imaging: an accurate method to evaluate arteriovenous malformations after stereotactic radiosurgery. J Neurosurg 85: 1044-1049, 1996

8) Pooh KH, Nakagawa Y, Jofuku N, Matsuura $H$, Fukuda K, Endo S, Matsumoto K: [A case of occult AVM diagnosed 17 years after subdural hematoma in the neonatal period]. No Shinkei Geka 24: 551-555, 1996 (Jpn, with Eng abstract)

9) Sonstein WJ, Kader A, Michelsen WJ, Llena JF, Hirano A, Casper C: Expression of vascular endothelial growth factor in pediatric and adult cerebral arteriovenous malformations: an immunocytochemical study. J Neurosurg 85: 838-845, 1996

10) Stein BM, Wolport SM: Arteriovenous malformations of the brain. Arch Neurol 37: 1-5, 1980

11) Waltimo O: The change in size of intracranial arteriovenous malformations. J Neurol Sci 19: 21-27, 1973

12) Watanabe E, Nagamune A, Matsuzawa H, Sano K: [A case of cerebral arteriovenous malformation (AVM) with presented remarkable enlargement during 13 years]. No To Shinkei 29: 1285-1289, 1977 (Jpn, with Eng abstract)

Address reprint requests to: T. Fuse, M.D., Department of Neurosurgery, National Tohsei Hospital, 762-1 Nagasawa, Shimizu-cho, Suntoh-gun, Shizuoka 411-8511, Japan. 